\title{
Computational modelling of the shot pattern of a sporting shotgun
}

\author{
R. Davidson, R. D. Thomson and A. E. Birkbeck \\ Ballistics and Impact Group, Department of Mechanical Engineering, University of Glasgow, UK
}

\begin{abstract}
There is an abundance of experimental data on the ballistic performance of sporting shotguns. However the successful development of computational models, that will help to reduce the commercial and environmental costs of extensive test programs, has been elusive. This paper describes the development and evaluation of a 3-D model that uses a commercial implementation of the distinct element method. The simulation gives realistic results for the effect of muzzle chokes on the spread of shot and provides evidence to support the well-known belief of practical shooters that a tapered constriction of 0.040 " $(1.02 \mathrm{~mm})$ in barrel diameter, the so-called 'full-choke', produces the optimum shot pattern. Detailed investigation of the internal ballistics of the shotgun shows that the reduction in diameter at the choke serves to compress the shot laterally as it exits the gun barrel. This affects the severity of interparticle collisions, creating a tighter, more uniform pattern. The accuracy of the computational results in the absence of air resistance tends to imply that the internal ballistics, rather than aerodynamic drag, dominates the shot pattern, although not the penetration power, over short ranges. Useful predictions of shotgun performance might then be obtained without resorting to full computational fluid dynamics simulation or to extensive field trials.
\end{abstract}

Units: Obsolescent British 'Imperial' units such as inches (") and ounces (oz.) and pounds (lb.) are still in widespread use for some of the critical dimensions and weights relating to firearms and ammunition, especially in legal definitions and in everyday speech. These units are retained in the text when appropriate. Equivalent ISO units are given.

Keywords: ballistics, computational modelling, distinct element analysis, shotguns

\section{Introduction}

The United Kingdom has some of the strictest firearms laws in the world. To prevent the carrying of concealable weapons, the Firearms (Amendment) Act (1997) (HMSO 1997) prohibited ownership of high calibre handguns, i.e firearms

Correspondence address:

R. D. Thomson, Department of Mechanical Engineering, University of Glasgow, Glasgow G12 8QQ, UK.

Tel.: +44 1413304313 , Fax: +44 1413304343 .

E-mail: rthomson@ mech.gla.ac.uk that are less than $60 \mathrm{~cm}$ in overall length or have a barrel length of less than $30 \mathrm{~cm}$, and with a chamber diameter of more than $0.22 "(5.59 \mathrm{~mm})$. Subsequently, the Firearms (Amendment) Act (1998) (HMSO 1998) prohibited small calibre $(0.22$ " or less) pistols. Shotguns are therefore one of the few types of firearm that can be legally held in the UK and then only with authorization from the police. This authorization takes the form of a 'Certificate to Hold Shotguns' as described in Section 2 of the Firearms Act (1968) (HMSO 1968) and is granted subject to the conditions prescribed by the Firearms Rules 1989 (HMSO 1989). 
Shotguns are used legally for vermin control, by farmers and licensed exterminators, and for sport. Other than deer, large game is uncommon in the UK and so shotguns are the most popular sporting firearm, both for game shooting and for clay target shooting. Participant numbers are difficult to gauge but estimates based on Clay Pigeon Shooting Association (CPSA) 1999 membership figures suggest that some 500000 people in the UK regularly shoot clays. The increasing popularity of the sport does however, present environmental problems, since the lead pellets most commonly used in shotgun cartridges are toxic and can seriously affect the reproduction, growth and ultimate survival of animals coming into accidental contact with them. There is also concern that the lead will leach into the soil and waterways and so enter the human food chain. There has therefore been an active search for nontoxic shot, iron and steel being the most promising candidates. The current situation is well-summarized in the proceedings of a seminar arranged by the British Association for Shooting and Conservation (BASC 1996). However further development is required if alternatives to lead are to be widely accepted, since these fail to deliver the effectiveness of lead pellet ammunition and can cause enhanced wear and damage to the weapon (Allsop 1996).

A large amount of experimental data has been gathered on the performance of shotguns over the long history of the weapon, but there is comparatively little information in the public domain, with Compton (1997) containing the most comprehensive review. A mathematical model of a shotgun, benchmarked against field trials and able to predict ballistic performance, remains elusive. Such a model would admit simulations and so reduce the commercial and environmental costs of extensive physical test programs, not only in the search for nontoxic shot but also, since shotguns inevitably figure in much firearms-related crime, in forensic pathology and in the science of wound ballistics (MacPherson 1994). Manual calculation of shotgun performance is impractical due to the number of interacting projectiles involved and so computational methods are required. Computational fluid dynamics (CFD) has been used as a tool for the study of the external ballistics phase (Leeming 2000), once the shot has left the barrel and air flow around and between adjacent particles may become significant, but it is perhaps less appropriate for the internal ballistics. Even finite element analysis is not well placed for the analysis of many discrete interacting projectiles and so is not particularly suitable for shotgun simulation. Distinct element analysis (DEA), as developed by Cundall \& Strack (1979), yields more realistic results and Birkbeck et al. (1998) describe what appears to be the first application of DEA to shotgun ballistics. In that 2-D model, the pellets were essentially cylinders in plane strain. However, the results gave confidence in DEA by computationally reproducing a situation that is well-known to practical shooters, i.e. that the traditional 'full-choke' (defined later) results in the optimum shot pattern, with degradation of the pattern resulting from either a decrease or an increase in the degree of choke. However that study did not compare the computational findings with detailed physical experiments. The current work extends the model to the more realistic 3-D case and attempts to benchmark the results against field trials. Initially, however, it is appropriate to review the construction and the principles of operation of sporting shotguns.

\section{The shotgun system: construction and operation}

The gun

As with many weapon systems, the design of shotguns has evolved incrementally during the history of shooting sports and their principles of operation have remained essentially the same for some 200 years. Figure 1 shows a general assembly of the working parts of a typical shotgun and of the firing mechanism in particular. When the gun is 'broken' open, by pivoting the steel barrel about the hinge pin, the hammer spring is compressed, forcing back the hammer and cocking the gun. A cartridge, comprising shot pellets, a percussion cap that acts as a priming charge, and the main 


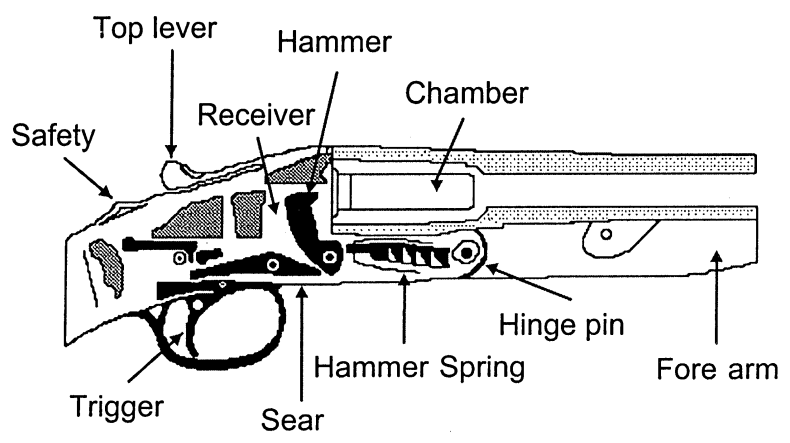

Figure 1 The shotgun firing mechanism.

propellant charge, is inserted into the chamber and the gun closed. Firing is initiated via the pulltrigger, which releases the hammer and allows it to strike and ignite the percussion cap. This subsequently ignites the main propellant charge.

The majority of modern sporting shotguns are of double-barrelled construction. This not only gives increased firepower but stiffens the barrel assembly and so improves the accuracy of the weapon. Barrels are positioned either 'side-by-side', the preferred configuration for game shooting, or one above the other, in the "over-and-under" configuration preferred for clay target shooting.

\section{The cartridge}

A cross-section of a typical shotgun cartridge is shown in Fig. 2. For the popular '12-bore' size of gun ('N-bore' is a barrel internal diameter that would be a snug fit on a lead ball weighing $1 / \mathrm{N}$ th of a pound), the cartridge is some $60 \mathrm{~mm}$ long and $20 \mathrm{~mm}$ in diameter. The cartridge assembly is enclosed in a stiff polymer outer case with a brass or brassed-steel base that retains the percussion cap and propellant and which expands when the propellant burns. This seals the receiver end of the chamber against the temperatures and pressures generated. The front of the case is crimped to contain the cartridge contents during storage and handling.

The spherical lead pellets or 'shot' are contained in a polymer cup that travels with the shot during the internal ballistics phase (when the shot is still

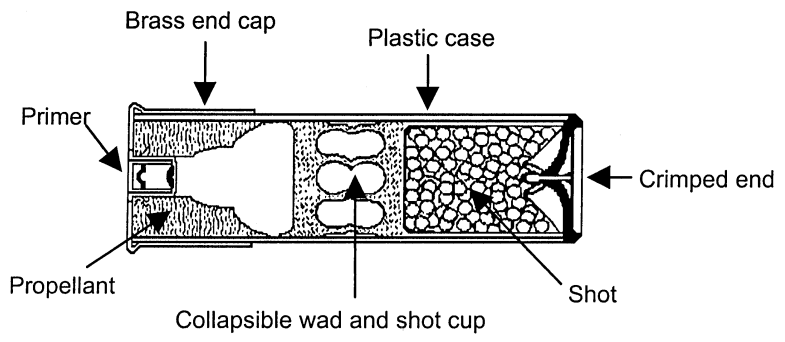

Figure 2 The cartridge construction.

inside the barrel) and for about $1 \mathrm{~m}$ of the external ballistics. This cup prevents the shot from rubbing on the inside of the barrel and reduces the 'flatspotting' that degrades performance and wears the barrel. The wad seals the barrel against the expanding gases that accelerate the cup during the internal ballistics and, nowadays, wad and cup are moulded as a single piece of soft polymer (Fig. 2 again). Joining the cup to the wad in the moulding are compliant pillars that buckle during the initial stages of firing, acting as shock-absorbers to reduce the peak recoil force on the shooter's shoulder.

The shot

Shot usually comprises small lead balls but, as noted previously, there is an active search for alternative materials. Shot is available in a variety of sizes and, obviously, cartridges of a particular size can hold a larger number of smaller pellets. Cartridges are, however, filled by weight and so the number of pellets can vary by some $20 \%$.

\section{Internal ballistics}

Internal or interior ballistics describes the time when the projectile is travelling inside the barrel. The internal ballistics of all firearms is governed by the nature of the propellant, the projectile and the geometry of the barrel. However, unlike most single-projectile small arms, shotguns are smoothbored, with no rifling on the inside of the barrel to impart a stabilizing rotation to the projectile. Shotgun barrels have five critical diameters that control the internal ballistics, as shown in Fig. 3. 


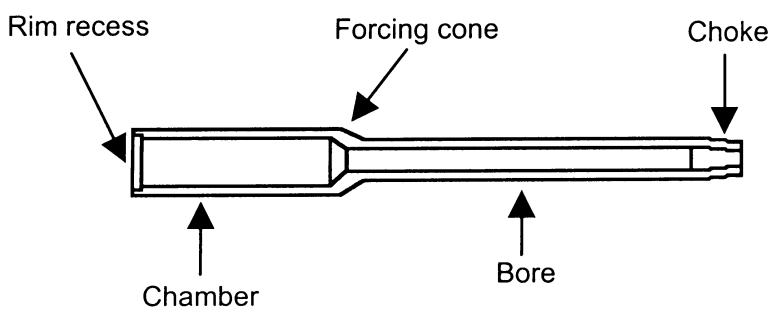

Figure 3 The barrel diameters.

Some of these are the basis of the legal definition of the weapon type and the Firearms Act (1968) (HMSO 1968) requires a 12-bore shotgun to have a barrel length greater than $24 "(610 \mathrm{~mm})$, with 26-28" being typical of sporting guns, a chamber diameter of $0.790 "(20.1 \mathrm{~mm})$ and a bore diameter of $0.722 "(18.3 \mathrm{~mm})$. These dimensions result in pressures of the order of $70 \mathrm{MPa}$ within the barrel after the main propellant charge has been ignited. As the gases expand, the wad-and-cup assembly and the contained shot are accelerated along the barrel up to a muzzle exit velocity of about $400 \mathrm{~m} \mathrm{~s}^{-1}$.

\section{External ballistics}

External or exterior ballistics describes the time from the projectile leaving the barrel until it nears the target. About $1 \mathrm{~m}$ into the external ballistics phase, air resistance causes the cup to petal along precut slits. This produces a rapid increase in drag and aerodynamic stall of the cup, which releases the shot. Once free of the cup, the shot pattern develops by the spread of the pellets, both in the direction of travel and in the transverse directions. While some spread is an essential feature of a shotgun, too much results in a drop in effectiveness.

The geometry of the shot pattern is described in terms of the shot string (its length) and the shot cone (its maximum diameter). 'Flat-spotted' pellets, that are permanently deformed during the internal ballistics, have an adverse effect on the shot string, their poorer aerodynamics causing them to fly more erratically and to fall behind undeformed pellets. This can cause the string length and diameter to increase beyond the optimum. However, even with undeformed pellets, the shot pattern increases in diameter during the flight and drag forces degrade the pellet velocity. All of this limits the effective range of shotguns to about $40 \mathrm{~m}$.

\section{The choke}

The design feature that most influences the external ballistics for a particular barrel is the choke, a slight taper in the final $40 \mathrm{~mm}$ of the barrel (Fig. 3 again). This lateral constriction slightly compresses the cup and its contained shot, just before they leave the barrel. This results in a reduction in the diameter of the shot cone and hence in the spread of shot at the target. Table 1 gives the dimensions of the various types of choke commonly used in modern shotguns. The choke is normally specified as an absolute value rather than as a proportion of the bore, which is related to the main barrel diameter.

The effects of choking the barrel are best observed with a distant target and, at about $35 \mathrm{~m}$, a fully choked barrel will deliver about $70 \%$ of the total shot in a 30 " $(760 \mathrm{~mm})$ pattern, a 'modified choke' about $65 \%$ and an 'improved cylinder' about $50 \%$. Each of these has its particular uses but practical experience has shown that the fully choked barrel produces the tightest shot pattern. Indeed, on the basis of 2-D simulations, Birkbeck et al. (1998) showed that an 'overchoked' barrel, that would never be used in actual practice, would indeed degrade the performance of the barrel.

\section{Field trials}

Field trials were carried out on private Universityowned farmland. These trials aimed to quantify the practical effect of a muzzle choke on the spread and penetration power of shot at various muzzle-

Table 1 Choke designations

\begin{tabular}{ll}
\hline Choke type & Constriction (") \\
\hline 'Full' & 0.04 \\
'Improved modified' or 'three-quarter' & 0.03 \\
'Modified' or 'half' & 0.02 \\
'Improved cylinder' or 'quarter' & 0.01 \\
'Cylinder' or 'skeet' & $0.00-0.01$ \\
\hline
\end{tabular}


to-target distances. The weapon used was a 12 bore (0.722", $18.5 \mathrm{~mm}$ diameter), double barrelled, side-by-side Baikal shotgun with 27\%" (702 mm) barrels. The right-hand barrel was a plain, unchoked cylinder while the left-hand had a fixed full choke.

Hull 'High Pheasant' cartridges were used, each containing a $1 \frac{1}{16}$ oz. $(30 \mathrm{~g})$ load of between 270 and 300 pellets of no. 6 shot. The cartridge case was $67 \mathrm{~mm}$ long overall, while the wad-and-cup was of one-piece, injection-moulded polymer construction.

The targets comprised $1 \mathrm{~m}$ squares of $3 \mathrm{~mm}-$ thick fibreboard, to monitor the spread of the shot, and $375 \times 300 \times 350 \mathrm{~mm}$ bundles of newsprint, to admit comparison of the depth of penetration. Targets were placed at ranges of 5, 10, 15, 20 and $25 \mathrm{~m}$ from the static firing point, these ranges being measured as muzzle-to-target distances. Four shots were fired at each range, one from each barrel into each type of target. An additional test was carried out with the newsprint at a range of $2 \mathrm{~m}$, in order to determine the penetration at a range close enough for the shot to be travelling almost at its muzzle velocity.

\section{Results and discussion}

Examination of the fibreboard targets showed that the shotgun pellets perforated the boards completely at all ranges, with considerable petalling around the exit holes. This made the spread of shot clearly visible. To quantify the spread of the shot at the various ranges, a 1-m square sheet of transparent acrylic polymer was used as a template. Eleven concentric circles, of diameter 50, 100, 200, $300 \ldots$ $1000 \mathrm{~mm}$ were scribed onto the surface of the sheet. This was placed over the perforated target and positioned such that the centre of the circles was near the centroid of the pattern of shot. The number of pellet holes within each circle was counted. This count was repeated for every target, to elicit the effect of the choke at each of the five ranges.

Almost all of the pellets from each cartridge struck and were detected in each target at up to $20 \mathrm{~m}$. However, many of the pellets missed the target completely at $25 \mathrm{~m}$ range and this result was discounted. The number of pellets enclosed within each diameter of circle, for both barrels, at test ranges of 10 and $20 \mathrm{~m}$, are shown in Fig. 4 (the results for 5 and $15 \mathrm{~m}$ are omitted for brevity but can be easily inferred). With more pellet strikes within any circular area, the fully choked barrel produced a consistently denser shot pattern at all ranges. This is summarized in Fig. 5, which shows the diameter of the circle enclosing $90 \%$ of the shot for both fully choked and cylinder barrels. The divergence between the two almost-straight lines shows that the benefit of the choke increases with range.

The penetrating power of the weapon was measured as the maximum depth at which
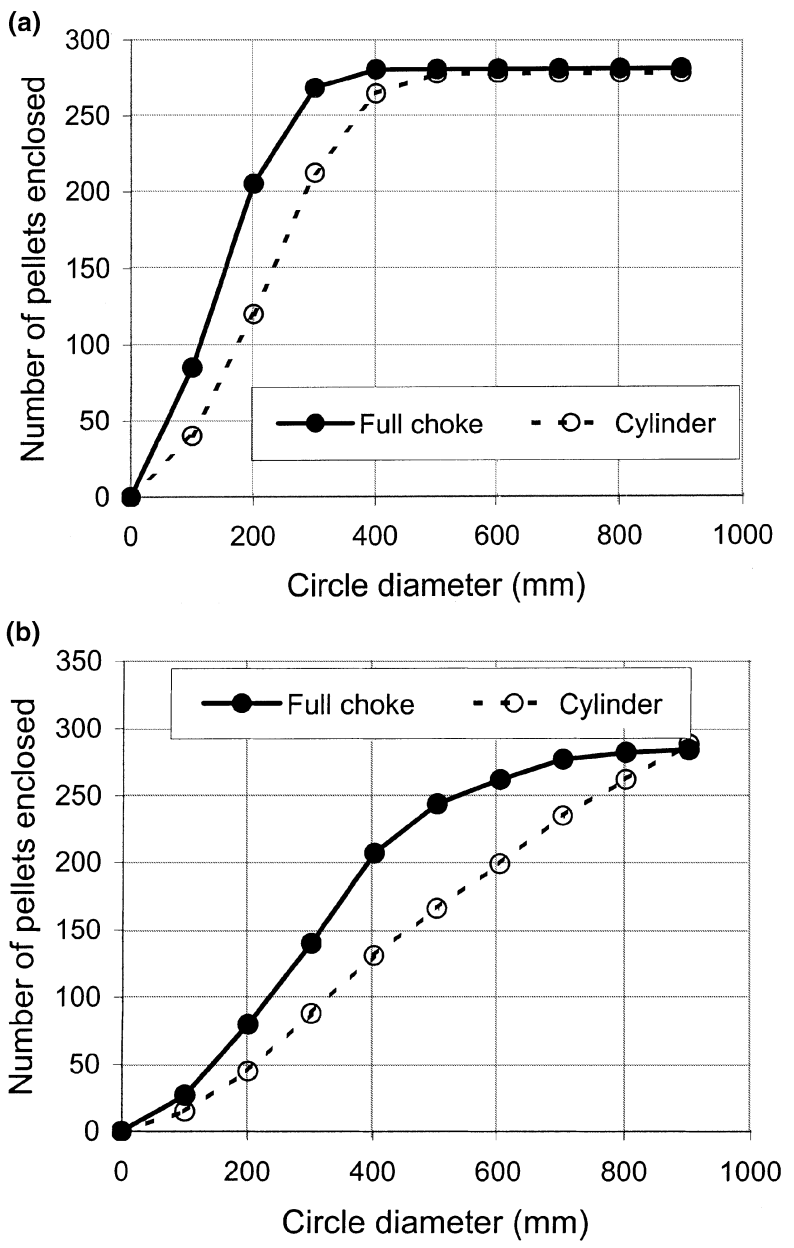

Figure 4 The experimentally measured spread of shot at (a) $10 \mathrm{~m}$ and (b) $20 \mathrm{~m}$ ranges. 


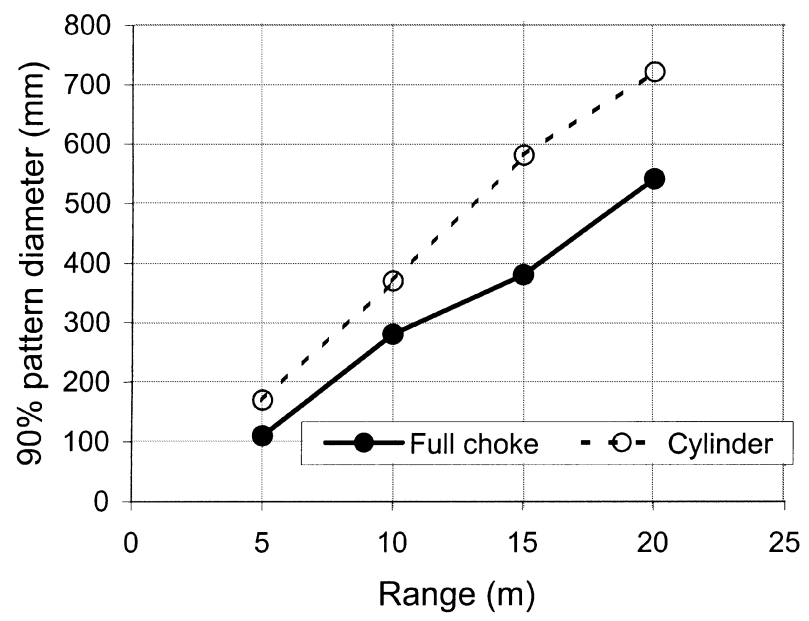

Figure 5 The effect of the choke on the spread of shot at each range.

perforation of any single sheet was seen in the newsprint block (Fig. 6). To ensure consistency of the sheet packing density, each damaged block was compressed by a $5-\mathrm{kg}$ mass placed on top of a level sheet of $6 \mathrm{~mm}$ plywood that completely covered the block. At very close range, the shot from both choked and unchoked barrels punched a single hole some $50 \mathrm{~mm}$ in diameter into the block. At such close range, the shot cone for both types of barrel is of small enough diameter for the target area to be subject to multiple strikes, with pellets at the leading edge of the shot string causing perforation damage that allows successive pellets to pass

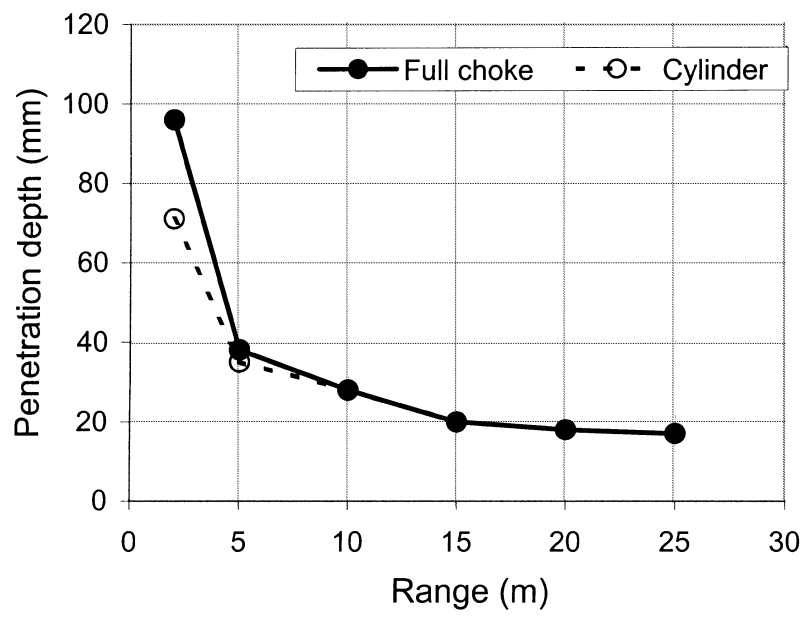

Figure 6 The effect of the choke on penetration at each range. though, initially unhindered, and to subsequently increase the overall depth of penetration. Even here, the effect of the choke is significant but the penetration power drops off very rapidly and, for ranges of $5 \mathrm{~m}$ and more, the pellets are sufficiently dispersed for the multiple-strike effect to disappear and only one pellet causes each penetration track. Beyond $5 \mathrm{~m}$, the choke has a reduced effect on penetration power (but not on the spread of shot) and it shows no improvement beyond $10 \mathrm{~m}$ range.

\section{Computational modelling}

\section{Distinct element modelling}

The DEA of Cundall \& Strack (1979) is based on the theory of a discontinuous medium that allows particles of the bulk material to slip and separate and to undergo unlimited displacements and rotations. This makes it particularly applicable to fragmentation problems. PFC ${ }^{3 \mathrm{D}}$ ('Particle Flow Code in 3-D') is a commercial 3-D DEA code (ITASCA Consulting Group Inc 1995). It was originally designed to aid in the engineering analysis of geomechanics problems by simulating the behaviour of particulate materials and, indeed, this remains the most common type of application (see, for example, te Kamp et al. (1998)). The work of the current authors appears to be the only application, so far, of DEA to weapon ballistics. In DEA, bulk material is assembled from spherical particles that may represent individual grains in the flow of sand or vehicles in a traffic stream or even pedestrians. The grains may be bonded together to model monolithic solids such as rock, concrete or steel, with elastic properties assigned to the bonds such that they mimic the macroscopic behaviour of the material. The fracture and fragmentation of the particles is simulated via progressive bond breaking under the applied load, the particles themselves being treated as rigid bodies.

For discontinuous systems with no initial bonding, such as the pellets in a shotgun cartridge, model generation involves the creation of a random assembly of particles, which are then compacted to 
the desired state of porosity. Birkbeck et al. (1998) thus simulated, in 2-D, the spread of shotgun pellets and their effect on a monolithic ceramic target that was modelled as a bonded assembly of particles. In the 3-D code, model boundaries are defined by surfaces that create a volume into which the spherical particles are placed. The particle assembly is compacted by expanding the radii of all the particles within the volumetric region and allowing them to move until they reach a state of equilibrium. Since all of the particles are expanded by the same amount, isotropy of the assembly is maintained. Physical and mechanical properties such as density and normal and shear stiffnesses can be assigned to the particles and walls. Boundary conditions such as velocity and force can also be assigned.

The solution algorithm uses an explicit timestepping scheme and, since time steps are of the order of microseconds, velocities and accelerations of particles and walls can be held constant within each step. At the start of each timestep, the model is scanned and the set of contacts is updated from the known positions of the particles and walls in the previous step. From the contact force, Newton's second law is applied to each particle to update the velocity and position. Wall positions are then updated, based on the specified wall velocities. Once a particle has assumed a velocity and direction, it continues on the same trajectory until it comes into contact with another entity. Models are generated, manipulated and analysed in $\mathrm{PFC}^{3 \mathrm{D}}$ using an internal, high-level programming language and graphical pre- and postprocessors are available.

\section{Distinct element analysis of the shotgun system}

\section{Geometry modelling}

$\mathrm{PFC}^{3 \mathrm{D}}$ does not admit curved walls and a circular cross-section such as a gun barrel must be modelled with facets. An octagonal cross-section was chosen as a compromise between accuracy and computational time on the heavily used Unix machine that was available (the elapsed time to simulate a $20-\mathrm{m}$ flight was $10 \mathrm{~h}$ ). Future studies on faster machines will use a more refined model. The barrel was modelled as an open ended facetted cylinder, with a length of $0.7 \mathrm{~m}$ and an across-flats diameter of $0.02 \mathrm{~m}$. For the fully choked simulation, the last $40 \mathrm{~mm}$ at the muzzle end of the barrel was tapered to reduce the diameter by $0.04 "(1.02 \mathrm{~mm})$. The cartridge cup was modelled with a cross section similar to that of the barrel but was initially closed at both ends. Within the cup, 220 discrete spherical elements were seeded to represent the a pellet fill of no. 6 game shot. These were packed by expanding the initial radii by a factor of 1.45 to gave a realistic porosity, but the total number of pellets is slightly less than in the physical test cartridges. This prevents prestraining of the purely elastic simulated pellets within the slightly reduced cross-section of the octagonal barrel.

\section{Boundary conditions}

On initial firing, real shot pellets are accelerated from behind by the cup-and-wad. This also compresses the shot in the barrel. However, acceleration cannot be directly assigned to walls in $\mathrm{PFC}^{3 \mathrm{D}}$ and uniform acceleration of the cup was approximated by increasing its velocity stepwise from 0 to $400 \mathrm{~m} \mathrm{~s}^{-1}$ in increments of $0.1 \mathrm{~m} \mathrm{~s}^{-1}$ over $4000 \times$ $1 \mu$ s timesteps. The cup walls were then held at $400 \mathrm{~m} \mathrm{~s}^{-1}$ for the remainder of the internal ballistics. In the fully choked model, the cup passes through the tapered region at the end of the barrel and is compressed laterally. In both models, upon leaving the barrel, the velocity of the cup was immediately reduced to $150 \mathrm{~m} \mathrm{~s}^{-1}$, allowing the shot to carry on ahead. The external ballistic effects of the expanding propellant gases propagating from the end of the barrel, and of air resistance, were not modelled.

\section{Terminal ballistics}

Terminal ballistics describes the final stage in the flight of a projectile to its target. To examine the effects of muzzle chokes on the terminal ballistics, 
the simulation was paused and the results inspected when the shot had travelled distances of 5, 10 and $20 \mathrm{~m}$. By viewing the output from the $\mathrm{PFC}^{3 \mathrm{D}}$ graphics postprocessor down the axis of the barrel, the proportion of shot contained within any given radius can be calculated, and the results compared with the physical experiments.

\section{Results and discussion}

Figure 7(a) shows a snapshot of the model cup in the barrel shortly after firing. The compression due to the acceleration of the cup during interior ballistics causes the shot to be initially compacted from the back and for an elastic wave to propagate through the shot. This causes some of the shot at the front of the cup to fly ahead of the main group. The separation of the cup from the shot shortly after leaving the muzzle allows the shot pattern to develop during the exterior ballistics, as in Fig. 7(b). There is only a slight increase in the diameter of the shot cone in the initial stages of flight which, since air resistance is not incorporated into the model, must be due to interparticle collisions. The collisions in the simulation are purely elastic and do not admit plastic deformation of the lead pellets. However, experiments in which the shot is fired but does not strike a solid target show that only a very small number of pellets are permanently deformed during the internal ballistics as, indeed, one would hope.

Figure 8 shows the proportion of the choked and unchoked shot contained within any given radius. The numbers of pellets are different in the simulation and the physical tests but the shape of the curves are in good in good agreement with the experimental results and in accord with practical

(a)

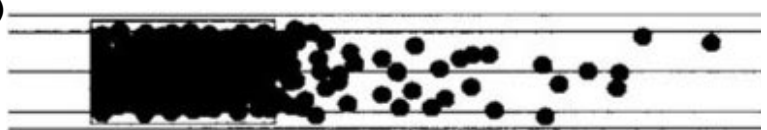

(b)

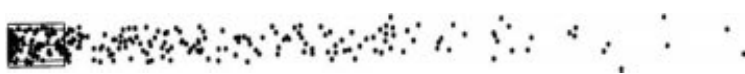

Figure 7 The computed shot pattern (a) shortly after firing and (b) after the cup has stalled. experience. Again, for brevity, only ranges of $10 \mathrm{~m}$ (Fig. 8a) and $20 \mathrm{~m}$ (Fig. 8b) are shown but the results for 5 and $15 \mathrm{~m}$ are easily inferred: the computational model predicts that at a range of $5 \mathrm{~m}$, both chokes would place the bulk of the shot within a $200-\mathrm{mm}$ circle and so would be effective against a clay target. This was confirmed by the field trials, in which the spread diameter for $90 \%$ of the shot at $5 \mathrm{~m}$ was $110 \mathrm{~mm}$ for a full-choke and $180 \mathrm{~mm}$ for a cylinder.

The effect of choke on the final pattern is best observed at distances above $5 \mathrm{~m}$. At a range of $10 \mathrm{~m}$, the fully choked barrel shows a significantly tighter pattern with better uniformity of shot
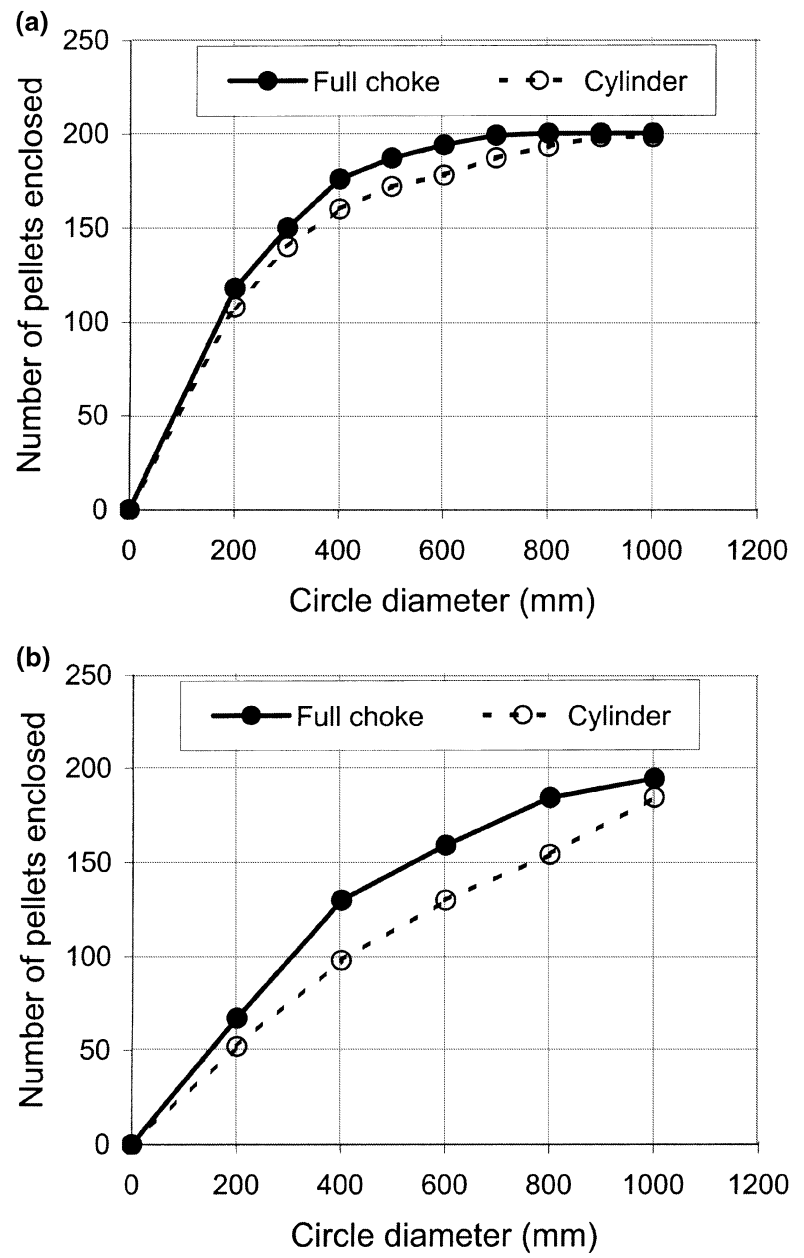

Figure 8 The computer-predicted spread of shot at (a) $10 \mathrm{~m}$ and (b) $20 \mathrm{~m}$ ranges.

Sports Engineering (2002) 5, 33-42 • 두 2002 Blackwell Science Ltd 
within the $200 \mathrm{~mm}$ circle. The cylinder barrel results in an uneven, 'blown' shot pattern, with more patchy, uncovered areas within the $200 \mathrm{~mm}$ circle. There is also an increase in the number of 'flyers', stray pellets outwith the main pattern area. The overall shot pattern diameter has increased to about $400 \mathrm{~mm}$ but with the full-choke creating a tighter, denser pattern within the smaller areas.

At $20 \mathrm{~m}$ range, the full-choke is even more effective in creating a tighter pattern, although the overall spread pattern increases to about $700 \mathrm{~mm}$ in diameter, again in agreement with the experimental results.

\section{The mechanics of muzzle chokes}

In spite of the large volume of experimental data available, the mechanism by which a muzzle choke modifies the shot pattern is not well understood and the optimum geometry of the full-choke for tight, uniform shot patterns has been the result of trial and error throughout the evolution of the shotgun. Understanding of the mechanics is aided by considering the interactions of the simulated particles during internal ballistics.

Figure 9 shows the radial distance, measured from the barrel axis for a particle initially lying near the centreline of the cup, plotted against the distance travelled down the barrel, for the fullchoke and unchoked barrel. The simulation yields

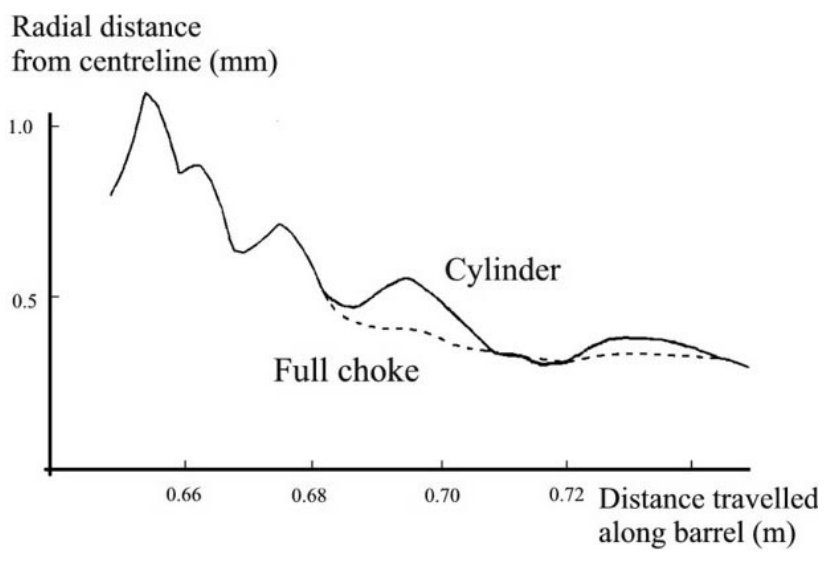

Figure 9 The radial motion of a particular pellet within the barrel. identical flight paths until the shot passes through the choke, just after $x=0.68 \mathrm{~m}$. Thereafter, the choked barrel produces more steady flight. This is possibly because the tapered constriction of the fully choked barrel compresses the shot laterally as it exits the muzzle and restricts lateral movement and the severity of elastic collisions. These cause most of the pellets on the outside of the shot string to diverge, increasing the pattern diameter at the target, which results in stray pellets that fly outwith the main shot pattern.

\section{Concluding remarks}

The field trials confirmed existing qualitative knowledge of the effect of muzzle chokes on the shot pattern and gave quantitative results on shot pattern and penetration power. They show that, for ranges above about $5 \mathrm{~m}$, the choke improves the shot pattern but not its penetration power. When the pattern was compared with a 3-D distinct element simulation, the results were found to be in surprisingly good agreement, given that air resistance was ignored. This suggests that elastic collisions of the shot during the internal ballistics are more important than the aerodynamics of the external ballistics in determining the shot spread, though obviously not the penetration power, at ranges up to $20 \mathrm{~m}$. Finally, it would be reasonable to infer that the spread of shot will also depend on the initial packing density of the cartridge, particularly with alternative materials. The DEA approach used here might then admit investigation of this with minimal need for expensive field trials.

\section{References}

Allsop, D.F. (1996) Damage caused to the choke of shot guns firing soft iron shot. In 'Non-Toxic Shot-Progress and Needs', BASC, Wrexham, UK.

BASC (1996) Non-toxic shot - progress and needs. Proceedings of a Seminar Held at RMCS Shrivenham. British Association for Shooting and Conservation, Wrexham, UK.

Birkbeck, A.E., Thomson, R.D. \& Wong, W.Y. (1998)

Distinct element simulation of the spread of shot from a 
sporting shotgun. In: 'The Engineering of Sport', ed. Haake, S.J. Blackwell Sciences, London.

Compton, D.J. (1997) An experimental and theoretical investigation of shot cloud ballistics. $\mathrm{PhD}$ Thesis, University of London.

Cundall, P.A. \& Strack, O.D.L. (1979) The development of constitutive laws for soil using the distinct element method. Numerical Methods in Geomechanics, 1, 289-317.

Farrar, C.L. \& Leeming, D.W. (1983) Military Ballistics - $a$ Basic Manual. Brassey's Defence Publishers, Oxford, UK. HMSO (1968) Firearms Act 1968. HMSO, London, UK.

HMSO (1989) Firearms Rules 1989. HMSO, London, UK. HMSO (1997) Firearms (Amendment) Act 1997. HMSO, London, UK.

HMSO (1998) Firearms (Amendment) Act 1998. HMSO, London, UK.
ITASCA Consulting Group Inc (1995) Particle Flow Code in 3 Dimensions $\left(\mathrm{PFC}^{3 \mathrm{D}}\right)$, Version 1.1 Users Manual, Minneapolis, Minnesota, USA.

te Kamp, L. Konietzky, H. \& Guérin, F. (1998) Modelling of the Chagan Underground Nuclear Test with the Distinct Element Method. International Fournal of Blasting and Fragmentation, 2, 295-312.

MacPherson, D. (1994). Bullet Penetration. Ballistic Publications, El Segundo, CA, USA.

Szmelter, J. \& Leeming, D.W. (2000) The aerodynamic interaction and dispersion of shotgun pellets at transonic velocities. Proceedings of the of the European Forum on Ballistics of Projectiles. 